\title{
M-estimation Methods for Fetal Heart Rate Estimation in Impulse Noises
}

\author{
Xiaoping Zeng, Yu Zhou, Jiao Tao, Junyi Yang and Shaohua Li \\ College of Communication Engineering, Chongqing University, Chongqing, \\ China \\ zxp@cqu.edu.cn
}

\begin{abstract}
Maternal electrocardiogram (MECG) suppression is a critical procedure to estimate fetal heart rate (FHR). The impulse noises in maternal abdomen signals (ADS) include electromyographic interference (EMGI) and fetal $R$ waves, which deteriorate FHR estimation in MECG suppression. In this paper, we introduce the M-estimation methods, a more robust methodology, to extract pure MECG in impulse noises. In order to evaluate the performance of M-estimation methods, two innovative quantitative performance metrics, namely loss degree of fetal $R$ wave and sensitivity of initial value, are proposed. Numerical results show that: (1) FHR estimation using M-estimation methods is more accurate than least square method (LSM) in impulse noises; (2) the robust performance of Fair is better than that of Cauchy in low SNR; (3) the robust performance of Cauchy is superior to that of Fair in high SNR.
\end{abstract}

Keywords: Maternal electrocardiogram (MECG) suppression, Fetal heart rate (FHR) estimation, M-estimation methods, Quantitative performance metrics

\section{Introduction}

Early information on fetal wellbeing throughout pregnancy and labor is of prime important for detecting the underlying defectives of the fetus heart. The most common method to acquire this information is though continuous monitoring of the fetal heart rate (FHR) [1]. The Doppler ultrasound detection is a conventional technique to monitor FHR. However, this technique requires highly trained personnel to operate [2] and it is considered as an invasive technique, especially, for long-time recordings [3]. The maternal abdomen signals (ADS) offer evident advantages over Doppler ultrasound. It is noninvasive by configuring electrodes on the maternal abdomen and simple to be operated even by pregnant women themselves. Nevertheless, FECG is strongly distorted by MECG of dominating energy $[4,5]$. Then, MECG is the most vital interference component for FHR estimation in ADS. To attain more accurate FHR estimation, entire MECG suppression from ADS is inevitable.

MECG suppression is considered in various processing methods [3, 5-7] to help extract fetal electrocardiogram (FECG) or FHR. A simple and convenient MECG suppression method was discussed by Matonia, et al., [5]. The method first extracts MECG by determination of maternal PQRST complex, then subtracts MECG from ADS to comply MECG suppression. However, the methods above do not consider the impact of impulse noises, such as electromyographic interference (EMGI) and fetal $\mathrm{R}$ waves. Due to the impulse characteristics of EMGI and fetal $\mathrm{R}$ waves [8], non-robust methods may recognize fetal $\mathrm{R}$ waves as MECG component, which causes fetal $\mathrm{R}$ wave loss after MECG suppression. Thus, M-estimation methods, a more robust methodology based on LSM, are introduced to execute MECG suppression in ADS. 
Furthermore, though quantitative analysis results are included in a few literatures [3, 9-12], the most widely used performance metrics are only signal-to-noise ratio (SNR) and SNR Improvement [11]. To enrich the quantitative performance metrics, two quantitative performance metrics are proposed. Synthetic and real ADS are processed for evaluation. The synthetic ADS consist of three components: EMGI, MECG and FECG. EMGI is generated by Laplace statistical model. FECG and MECG are generated by a simplified model. Real ADS from the database [13] are used to verify the practical application of M-estimation methods.

The remainder of the paper is organized as follows. In Section 2, M-estimation theory is reviewed first, modeling process for ADS and proposed performance metrics are described then. Results and discussion are presented in Section 3. Finally, conclusion on M-estimation methods for FHR estimation in impulse noises is given in Section 4.

\section{Methods}

\subsection{M-estimation Theory}

As a category of generalized estimation, M-estimation methods are expressed as $[14,15]$ :

$$
\hat{X}_{j}=\arg \min \sum_{i=1}^{N} \rho\left(Y_{i}-A X_{i}\right), 1 \leq i \leq N, 1 \leq j \leq N
$$

Where $\hat{X}_{j}$ is the estimated result of the unknown interested signal of $X_{i}, A$ is a coefficient matrix, $Y_{i}$ is the observed signal, $\rho(\cdot)$ is a criterion function concerned with parameter $\left(Y_{i}-A X_{i}\right)$. In fact, it is very difficult to obtain $\hat{X}_{j}$ from Eq. (1). The simplified expression of M-estimation is generally showed as Eq. (2).

$$
\sum_{i=1}^{N} \varphi\left(Y_{i}-A X_{i}\right)=0,1 \leq i \leq N
$$

Where $\varphi(\cdot)$ is the differentiation of $\rho(\cdot)$. The above is similar with the form of LSM. The modified expression of M-estimation methods performs more effectively in Eq. (3), which is an equivalent weight model like LSM, and where $\bar{P}=P \omega, P$ is the weight coefficient and $\omega$ is the weight factor. The derivation process of the equivalent weight model is described by Zhou et al [15]. The extremal function $\varphi(\cdot)$ and weight factor function $\omega(\cdot)$ of M-estimation methods used in this paper are shown in Table 1.

$$
\hat{X}=\left(A^{T} \bar{P} A\right)^{-1} A^{T} \bar{P} Y
$$

\section{Table 1. The Extremal functions $\varphi(\cdot)$ and Weight functions $w(\cdot)$ of Different} Methods

\begin{tabular}{lcc}
\hline Method & $\varphi(\cdot)$ & $w(\cdot)$ \\
\hline Huber & $\varphi(Z)= \begin{cases}Z, & |Z| \leq k \\
k \cdot \operatorname{sign}(Z) . & |Z|>k\end{cases}$ & $w(Z)= \begin{cases}1, & |Z| \leq k \\
k / Z \mid . & |Z|>k\end{cases}$ \\
Fair & $2 Z /(1+Z / k)$ & $2 /(1+Z / k)$ \\
Cauchy & $Z /\left(1+Z^{2}\right)$ & $1 /\left(1+Z^{2}\right)$ \\
LSM & $Z$ & 1 \\
\hline
\end{tabular}

Where $k$ is the threshold value of residual error $Z\left(Z_{i}=Y_{i}-A X_{i}\right)$. Mestimation methods would be well performed in robustness when $k$ is set as the value in an appropriate range. Thus, the exact values of $k$ for different $\mathrm{M}$ - 
estimation methods are determined as bellow: $k=1.345$ for Huber, $k=2.385$ for Cauchy, and $k=1.400$ for Fair. As Table 1 shows, the extremal function and weight function of Huber are divided into two parts based on the value of $k$.

The value of the weight is crucial for the robustness in each method. Iterations should be constantly executed to find the optimum value of the weight. Given the value of $k$, the procedure of iterations for M-estimation methods can be depicted as:

Step 1: set initial values of MECG model as Figure 2

Step 2: extract MECG using M-estimation methods

Step 3: choose a weight $P$ corresponding to the step length

Step 4: substitute the weight $P$ in equivalent weight model

Step 5: calculate a new residual error, and the iteration time increases by 1

Step 6: repeat step 3 to step 5 until the iteration time reaches $10^{3}$ or $Z$ reaches $10^{-6}$

Step 7: output M-estimation parameters

\subsection{Modeling and Initial Value Determination}

2.2.1. Real ADS Modeling: Synthetic ADS are used to validate the performance of M-estimation methods first. The most widely-used three-dimensional (3-D) dynamic model with coordinates $(x, y, z)$ is first proposed by McSharry [16]. Based on the 3D model, a more simplified model shown as Equation 4 is proposed by Sayadi for variable $z$, which indicates that one ECG beat could be formulated as a sum of five Gaussians [11]. In this paper, we use the classical model to synthesize MECG and FECG.

$$
z\left(\alpha_{i}, b_{i}, \theta_{i}\right)=\sum_{i \in\{P, Q, R, S, T\}} \alpha_{i} \exp \left(-\frac{\Delta \theta_{i}^{2}}{2 b_{i}^{2}}\right)
$$
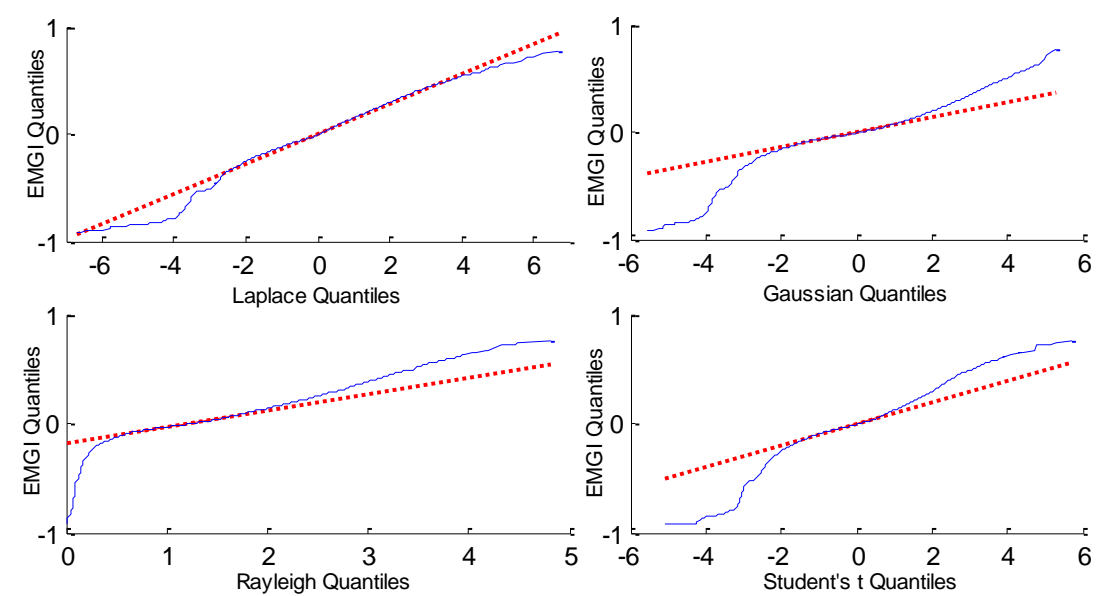

Figure 1. QQ-plot Comparative Results between EMGI and Laplace, Gaussian, Rayleigh and Student's t Distributions

In fact, EMGI is impulse noise in real ADS [13]. A suitable model should be chosen for it to synthesize ADS. However, the random characteristics of EMGI cause difficulties in modeling with their waveform morphology. In view of this, an approximate statistical model is chosen for EMGI.

The qq-plot comparative results between EMGI and four heavy-tailed distributions are displayed in Figure 1. The mean and standard deviation both for Laplace and Gaussian distributions are 0 and 1 respectively; the standard deviation of the Rayleigh distribution is 1 , and the Student's t distribution is with 20 degrees of 
freedom. Figure 1 shows that the distribution of EMGI is more similar to Laplace distribution. Therefore, Laplace distribution is chosen to model EMGI in simulation.

2.2.2 Determining the Initial Value of MECG Model and the Threshold of Residual errors: Since M-estimation methods are batch-processed and model-based, all the data to be processed and initial parameters of the model are required at first. The framework in Figure 2 is proposed to determine the initial values of one MECG beat.

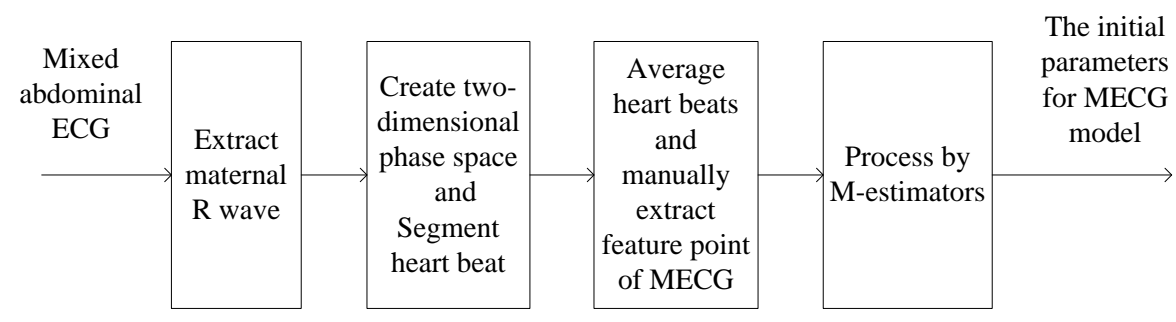

Figure 2. The Process of Determining the Initial Values of MECG

Five-point differential threshold [17] with less computation is used to detect $\mathrm{R}$ wave in ADS. The method proposed by Clifford [18] is utilized to obtain average heartbeat. The initial parameters for amplitude and angular position for the average heartbeat, namely $\alpha_{i}(i=P, Q, R, S, T)$ and $\theta_{i}(i=P, Q, R, S, T)$, are set as empirical values, and width $b_{i}(i=P, Q, R, S, T)$ were all presupposed as 0.04 second.

\subsection{Performance Metrics for Evaluation}

As a common performance metric to evaluate estimation accuracy of methods, SNR improvement of output signal given by Eq. (5) $[11,19]$ is used in this paper.

$$
S N R_{i m p}=10 \lg \sum_{i}\left(x_{n}(i)-x_{c}(i)\right)^{2} / \lg \sum_{i}\left(x_{d}(i)-x_{c}(i)\right)^{2}
$$

Where $x_{c}(i)$ is pure MECG, $x_{n}(i)$ represents input ADS with impulse noises, and $x_{d}(i)$ is MECG extracted by different methods. Change the SNR of input synthetic ADS from $-5 \mathrm{~dB}$ to $7 \mathrm{~dB}$, the corresponding SNR improvements are displayed in Figure 5. However, this metric only evaluates the estimation accuracy of MECG. The error caused by erroneous MECG separation can't be calculated. Then the loss degree of fetal $\mathrm{R}$ wave is proposed to calculate the error. Besides, the sensitivity of initial value is proposed to evaluate the sensitive extent for different M-estimation methods.

2.3.1 Loss Degree of Fetal $\mathbf{R}$ Wave: the loss degree of fetal $R$ wave is proposed to evaluate the loss extent of amplitude and angular position for $\mathrm{R}$ wave after MECG suppression, which is given by

$$
\text { Loss }=\frac{1}{N} \sum_{i=1}^{N}\left|x_{\text {out }}(i)-x_{p}(i)\right| /\left|x_{p}(i)\right|
$$

Where $x_{p}$ and $x_{\text {out }}$ represent $\mathrm{R}$ wave amplitude of pure and estimated FECG, respectively. This metric can calculate the departure between estimated and real amplitude of fetal $\mathrm{R}$ wave.

The synthesis ADS used for quantitative analysis in Figure 6 and Figure 7 only contained pure MECG and FECG, where FECG was regarded as impulse interference. 
However, the maternal-fetal heart rate ratio (HRR) and amplitude ratio (AR) are not always the same for pregnant during the whole gestation. In view of this, different maternal-fetal AR and HRR were considered in Figure 6 and Figure 7. Maternal-fetal AR was changed from 3 to 7 when maternal-fetal HRR was kept as 2.1 in Figure 6. Figure 7 displayed the case where maternal-fetal HRR range changes from 1.5 to 2.3 when maternal-fetal AR is kept as 4.

2.3.2 Sensitivity of Initial Value: The initial parameters obtained in Figure 2 were not accurate for all heartbeats. To evaluate the impact of inaccurate initial parameter on MECG extraction of three M-estimation methods, the sensitivities to angular position $(\theta)$ and amplitude $(\alpha)$, were discussed with the metric:

$$
A(\cdot)=L_{M}(\cdot) / L_{L S M}(\cdot)
$$

Where $A(\cdot)$ denotes the loss degree, $L_{L S M}(\cdot)$ and $L_{M}(\cdot)$ represent the range of model parameters when the loss extent of fetal $\mathrm{R}$ wave exceeds $85 \%$ using LSM and different M-estimation methods, respectively. The formula shows that $A(\cdot)$ is relatively smaller if the method is not sensitive to the initial parameter of the model.

In Table 2, several typical waveform segments were synthesized, which included superposed maternal-fetal PR waves, superposed maternal-fetal TR waves and superposed maternal-fetal RR peaks.

\section{Results and Discussion}

The real ECG data used in this paper is from Department of obstetrics of Silesia medical university, which is released recently [13]. Besides maternal ECG, synchronous fetal scalp ECG is available in this database. Using the simplified model in Equation (4), MECG and FECG were generated by fitting real ADS and real fetal scalp ECG in the database, respectively. The sampling rate was assigned as $1000 \mathrm{~Hz}$. The performances of four methods were analyzed by comparing three metrics, namely output SNR improvement, loss degree of fetal $\mathrm{R}$ wave, and sensitivity of initial value.

\subsection{Quantitative Analysis for Synthetic ADS}

The synthetic ADS includes MECG, FECG and EMGI in Figure 3, where FECG and EMGI are regarded as impulse noises. The input SNR is $6 \mathrm{~dB}$, which is more common in the perinatal period. It can be seen from the figure that three $\mathrm{M}$ estimation methods can successfully extract MECG without the interference of impulse noises. LSM has the worst performance, which has a devastating influence on subsequent result of MECG suppression shown in Figure 4. It also displays that M-estimation methods can extract pure MECG when fetal $\mathrm{R}$ waves overlaps maternal $\mathrm{P}$ waves and $\mathrm{T}$ waves, while all the four methods become invalid in solid circle where maternal-fetal RR peaks overlap. 


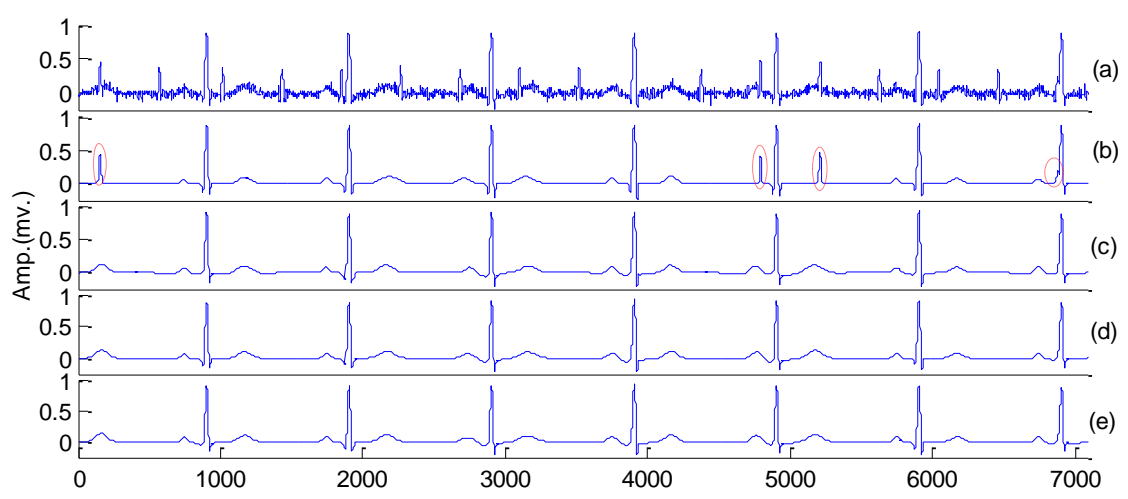

Figure 3. Maternal ECG Signals Extracted by Four Methods ( $a$ is Synthetic ECG, and b, c, d, e are Respectively by LSM, Huber, Cauchy, Fair)

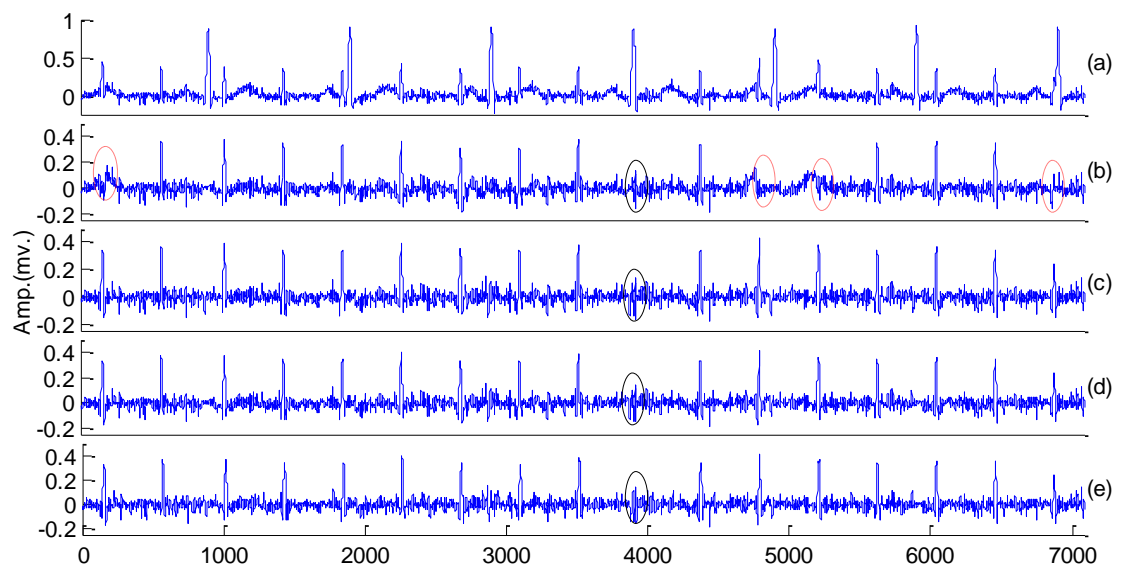

Figure 4. Noisy FECG Signals Obtained by Four Methods (a is Synthetic ECG, and b, c, d, e are Respectively by LSM, Huber, Cauchy, Fair)

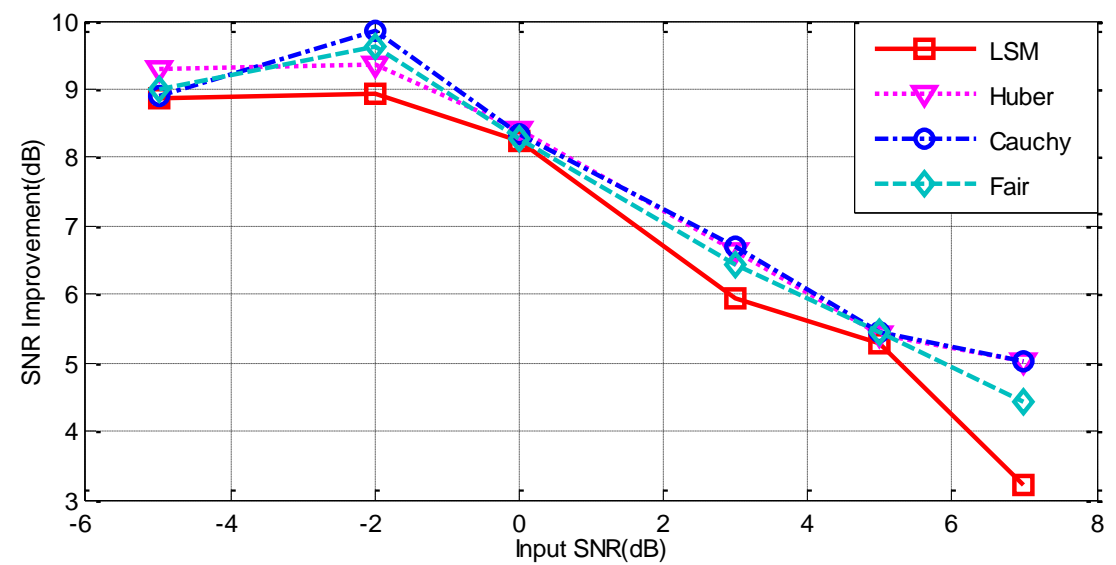

Figure 5. SNR Improvements with Different Input SNR

SNR improvements with different input SNR are displayed in Figure 5. It demonstrates that the performances of Cauchy and Huber are better when the input SNR is relatively higher, $6 \mathrm{~dB}$ for example. The SNR improvement of Huber and Fair is relatively stable when the input SNR is relatively lower, $-2 \mathrm{~dB}$ to $-5 \mathrm{~dB}$ for instance. It proves that Huber is the most stable method in impulse noises. Cauchy have better robust performance than Fair in high SNR, while Fair have greater robust capability than Cauchy in low SNR. 
Four methods were used to fit wave forms with different maternal-fetal AR and HRR when input SNR is around $10 \mathrm{~dB}$ (high SNR), and corresponding results are displayed in Figure 6 and Figure 7. As Figure 6 displays that the loss degree of fetal $\mathrm{R}$ wave using LSM is almost two times as great as that of M-estimation methods. Figure 7 shows loss degrees of different maternal-fetal HRR. The loss caused by LSM is greater by $25 \%$, which is nearly two times greater than that caused by Cauchy.

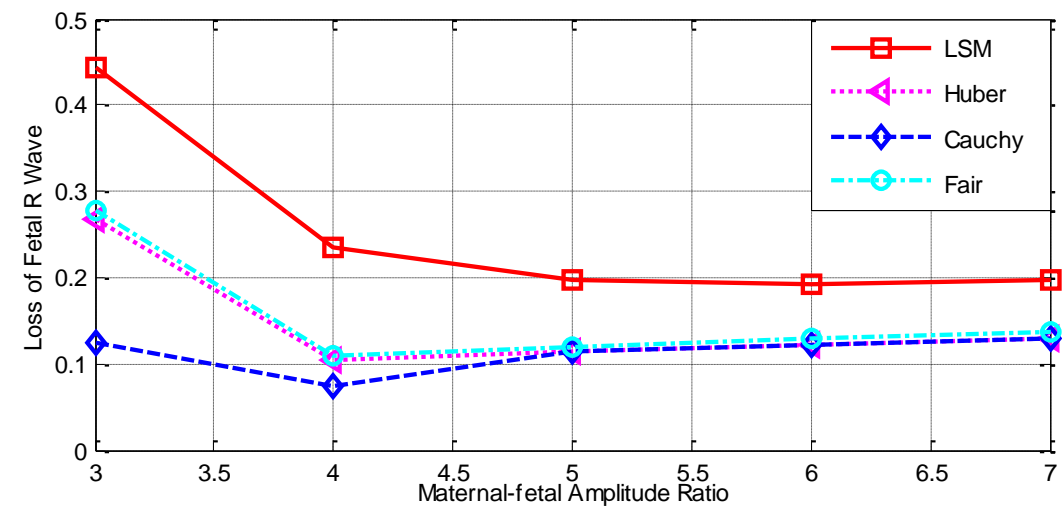

Figure 6. Loss of FECG's R-peak with different maternal-fetal AR

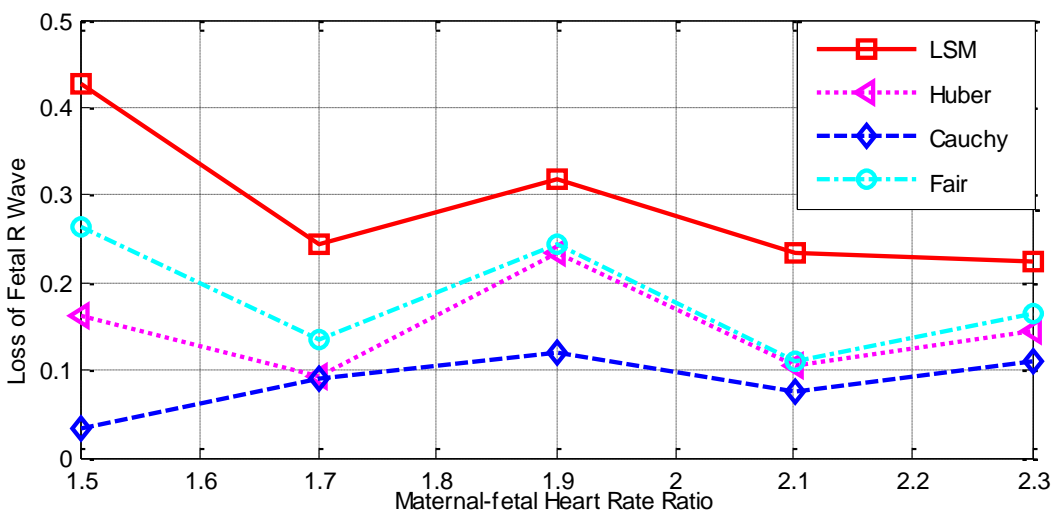

Figure 7. Loss of FECG's R-peak with Different HRR

Table 2. Loss Degree of Fetal R Wave for Different M-estimation Methods

\begin{tabular}{|c|c|c|c|c|}
\hline loss degree & Methods & Maternal-fetal PR & Maternal-fetal TR & Maternal-fetal RR \\
\hline \multirow{3}{*}{$A_{\alpha}$} & Huber & $11.62 \%$ & $3.86 \%$ & \multirow{2}{*}{$100 \%$} \\
\cline { 2 - 5 } & Cauchy & $10.93 \%$ & $3.71 \%$ & \\
\cline { 2 - 5 } & Fair & $13.84 \%$ & $5.27 \%$ & \multirow{2}{*}{$100 \%$} \\
\hline \multirow{3}{*}{$A_{\theta}$} & Huber & $21.35 \%$ & $13.75 \%$ & \\
\cline { 2 - 5 } & Cauchy & $21.02 \%$ & $13.67 \%$ & \\
\cline { 2 - 5 } & Fair & $22.15 \%$ & $14.19 \%$ & \\
\hline
\end{tabular}

As shown in Table 2, the input SNR is also kept as $8 \mathrm{~dB}$ (high SNR). Three Mestimation methods all have lower sensitivity to amplitude than angular position, and Cauchy is the robustest to initial value. Besides, all the methods, especially Cauchy, have good robust performance when fetal $\mathrm{R}$ waves overlap maternal $\mathrm{P}$ waves and $\mathrm{T}$ waves. However, all the three methods fail to eliminate the interference of the fetal $\mathrm{R}$ wave in case of superposed maternal-fetal RR peaks. 


\subsection{Detection for Real ECG}

A part of real ADS r_04 (channel 5) from [13], lasting for 7 seconds, was used for illustration. Notice that baseline drift, High-frequency noise, and power interference are present in real ECG. The real ADS are preprocessed by double median filter and notch filter for $60 \mathrm{~Hz}$. Set initial parameters of model as Figure 2, then the extracted MECGs from ADS and the remaining FECGs are respectively displayed in Figure 8 and Figure 9. Two dashed circles mark up the FECG estimated incorrectly using LSM. After MECG suppression, the FECGs with noises are shown in Figure 9. The locations in dashed circles using LSM all lose FECG. Solid circles mark up the location where maternal-fetal RR peaks overlap. As discussed in Subsection 3.1, four methods extract MECG with FECG, which causes the loss of fetal R wave in Figure 9.

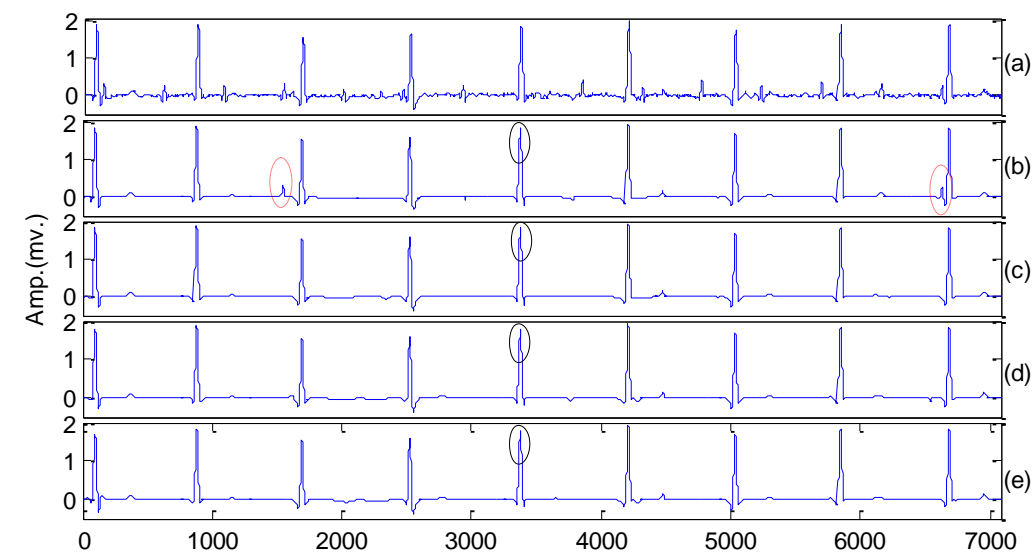

Figure 8. Maternal ECG Signals Extracted by Four Methods (a is Real ADS, and b, c, d, e are Respectively by LSM, Huber, Cauchy, Fair)

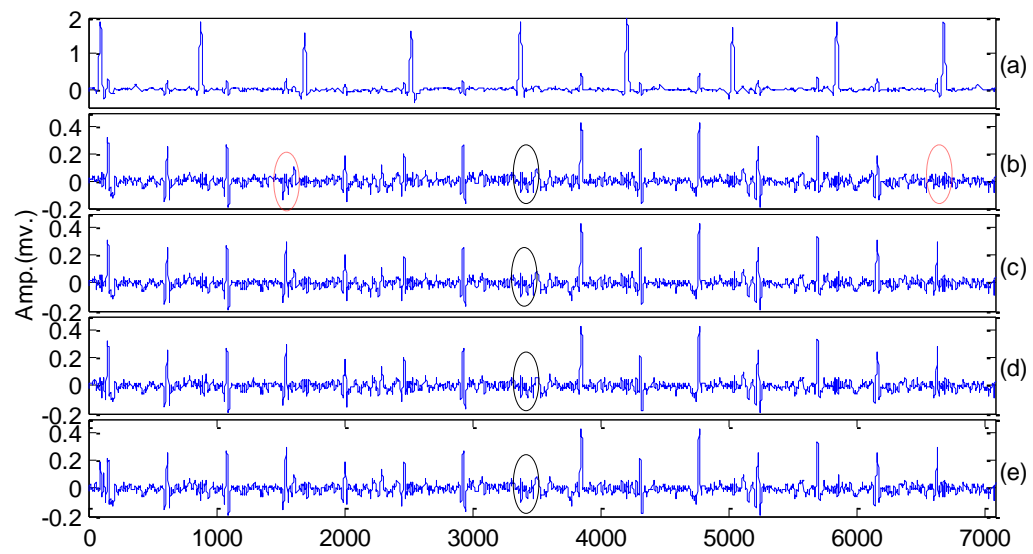

Figure 9. Noisy FECG Signals Obtained by Four Methods (a is Real ADS, and b, c, d, e are Respectively by LSM, Huber, Cauchy, Fair)

Intercepted parts of different ADS (r_01, r_04, r_07 and $r_{-} 08$ ) lasting for 11 seconds, the number of real $\mathrm{R}$ waves, identified fetal $\mathrm{R}$ wave numbers and determination accuracy of fetal $\mathrm{R}$ waves using different methods are recorded in Table 3. Among the four ADS, the SNR of $r_{-} 01$ is the lowest, where the accuracy of Huber and Fair is more obvious. Superposed maternal-fetal RR peaks are not included in $r_{-}$08, where M-estimation methods find out all fetal $R$ peaks successfully. The SNR of $r_{-} 04$ and $r_{-} 07$ is relatively high, where the accuracy of Cauchy is higher than that of Fair. 


\section{Table 3. Fetal R Wave Numbers and Determination Accuracy using} Different Methods

\begin{tabular}{c|cc|cc|cc|cc}
\hline & \multicolumn{2}{|c|}{ r_01 } & \multicolumn{2}{c|}{ r_04 } & \multicolumn{2}{c|}{ r_07 } & \multicolumn{2}{c}{ r_08 } \\
& numbers & accuracy & numbers & accuracy & numbers & accuracy & numbers & accuracy \\
\hline Real & 24 & 0 & 24 & 0 & 23 & 0 & 23 & 0 \\
LSM & 17 & $70.83 \%$ & 21 & $87.50 \%$ & 17 & $73.91 \%$ & 21 & $91.30 \%$ \\
Huber & 21 & $87.50 \%$ & 23 & $95.83 \%$ & 21 & $91.30 \%$ & 23 & $100 \%$ \\
Cauchy & 19 & $79.15 \%$ & 23 & $95.83 \%$ & 21 & $91.30 \%$ & 23 & $100 \%$ \\
Fair & 21 & $87.50 \%$ & 22 & $91.67 \%$ & 20 & $86.96 \%$ & 23 & $100 \%$ \\
\hline
\end{tabular}

\section{Conclusion}

One of the main contributions of this paper is successfully introducing M-estimation methods to suppress MECG from ADS in impulse noises for FHR estimation. In addition, two innovative metrics are proposed to verify the performance of different methods, loss degree of fetal $R$ wave is accuracy evaluation of fetal $R$ wave estimation, and sensitivity of initial value analyzes the sensitive characteristics of M-estimation methods. This work certificates that M-estimation methods have greater robust capability than LSM in impulse noises. Moreover, it compares the performance of three classical M-estimation methods by utilizing three metrics. The conclusions are: (1) among the M-estimation methods, the robust performance of Huber is most reliable and most stable; (2) the robust performance of Fair is better than that of Cauchy in low SNR; (3) the robust performance of Cauchy is superior to that of Fair in high SNR. Furthermore, M-estimation Methods for FHR Estimation can be improved further to extract pure MECG in case of superposed maternal-fetal RR peaks.

\section{Acknowledgements}

This work was supported by the National Natural Science Foundation of China (NSFC) under Grants 61171089 and 61302054, the Natural Science Foundation Project of Chongqing (cstc2012jjA40056, cstc2012jjB40010). The authors would like to thank the anonymous referees whose insightful comments helped us to improve the presentation of the paper.

\section{References}

[1]. D. Devane, J. G. Lalor, S. Daly, W. McGuire, and V. Smith, "Cardiotocography versus intermittent auscultation of fetal heart on admission to labour ward for assessment of fetal wellbeing", Cochrane Database Syst Rev, vol. 2 (2012).

[2]. E. Karvounis, M. Tsipouras, C. Papaloukas, D. Tsalikakis, K. Naka, and D. Fotiadis, "A non-invasive methodology for fetal monitoring during pregnancy", Methods of information in medicine, vol. 49, (2010), pp. 238.

[3]. E. C. Karvounis, M. G. Tsipouras, D. I. Fotiadis, and K. K. Naka, "An automated methodology for fetal heart rate extraction from the abdominal electrocardiogram", Information Technology in Biomedicine, IEEE Transactions on, vol. 11, (2007), pp. 628-638.

[4]. E. Karvounis, C. Papaloukas, D. Fotiadis, and L. Michalis, "Fetal heart rate extraction from composite maternal ECG using complex continuous wavelet transform", Computers in Cardiology, (2004), pp. 737740 .

[5]. A. Matonia, J. Jezewski, K. Horoba, A. Gacek, and P. Labaj, "The maternal ECG suppression algorithm for efficient extraction of the fetal ECG from abdominal signal", Engineering in Medicine and Biology Society, 2006. EMBS'06, 28th Annual International Conference of the IEEE, (2006), pp. 3106-3109.

[6]. M. I. Ibrahimy, F. Ahmed, M. A. M. Ali, and E. Zahedi, "Real-time signal processing for fetal heart rate monitoring", Biomedical Engineering, IEEE Transactions on, vol. 50, (2003), pp. 258-261.

[7]. R. Martin-Clemente, J. L. Camargo-Olivares, S. Hornillo-Mellado, M. Elena, and I. Roman, "Fast technique for noninvasive fetal ECG extraction", IEEE transactions on bio-medical engineering, vol. 58, (2011), pp. 227-230.

[8]. G. Li, X. Zeng, X. Zhou, Y. Zhou, G. Liu, and X. Zhou, "Robust suppression of nonstationary powerline interference in electrocardiogram signals", Physiological measurement, vol. 33, (2012), pp. 1151. 
[9]. S. Wu, Y. Shen, Z. Zhou, L. Lin, Y. Zeng, and X. Gao, "Research of fetal ECG extraction using wavelet analysis and adaptive filtering", Computers in biology and medicine, vol. 43, (2013), pp. 1622-1627.

[10].S. Kharabian, M. B. Shamsollahi, and R. Sameni, "Fetal R-wave detection from multichannel abdominal ECG recordings in low SNR", Engineering in Medicine and Biology Society, 2009, EMBC 2009, Annual International Conference of the IEEE, (2009), pp. 344-347.

[11].O. Sayadi and M. B. Shamsollahi, "ECG Denoising and Compression Using a Modified Extended Kalman Filter Structure", Biomedical Engineering, IEEE Transactions on, vol. 55, (2008), pp. 22402248.

[12].R. Sameni, M. B. Shamsollahi, C. Jutten, and G. D. Clifford, "A nonlinear Bayesian filtering framework for ECG denoising", Biomedical Engineering, IEEE Transactions on, vol. 54, (2007), pp. 2172-2185.

[13].Information on:http://physionet.org/physiobank/database/adfecgdb/ [Online].

[14].Y. Zou, S.-C. Chan, and T.-S. Ng, "Least mean M-estimate algorithms for robust adaptive filtering in impulse noise", Circuits and Systems II: Analog and Digital Signal Processing, IEEE Transactions on, vol. 47, (2000), pp. 1564-1569.

[15].J. Zhou, Y. Huang, and Y. Yang, "Robust least squares method", Huazhong University of science and Technology Press (1997).

[16].P. E. McSharry, G. D. Clifford, L. Tarassenko, and L. A. Smith, "A Dynamic Model for Generating Synthetic Electrocardiogram Signals", IEEE Trans Biomed Eng., vol. 50, (2003), pp. 289-294.

[17].O. Sayadi and M. B. Shamsollahi, "Model-based fiducial points extraction for baseline wandered electrocardiograms", Biomedical Engineering, IEEE Transactions on, vol. 55, (2008), pp. 347-351.

[18].G. Clifford, A. Shoeb, P. McSharry, and B. Janz, "Model-based filtering, compression and classification of the ECG", International Journal of Bioelectromagnetism, vol. 7, (2005), pp. 158-161.

[19].O. Sayadi, M. B. Shamsollahi, and G. D. Clifford, "Synthetic ECG generation and Bayesian filtering using a Gaussian wave-based dynamical model", Physiological measurement, vol. 31, (2010), pp. 1309.

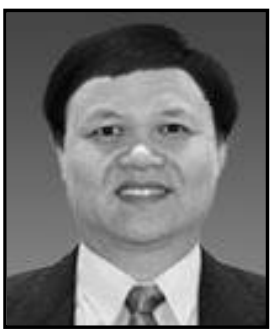

XiaoPing Zeng, Received his $\mathrm{BS}$, MS and $\mathrm{PhD}$ from Chongqing University in 1982, 1987 and 1996, respectively. $\mathrm{He}$ is currently a Professor of College of Communication Engineering at Chongqing University. His research interests include Neural network theory and its applications, information and signal processing, Broadband wireless communications.

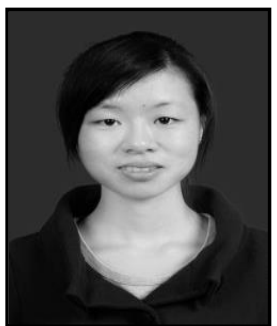

Yu Zhou, Received her B.E. degree from Chongqing University, Chongqing, China in 2013. She is a master student at the College of Communication Engineering, Chongqing University, China. Her research interests include biomedical signal processing and data analysis. 